\title{
Prevalência e características do tabagismo na população universitária da região de Lins-SP
}

Prevalence and smoking characteristics in the university population of the region of Lins-SP

Predominio y características del tabaquismo en la población universitaria de la región de Lins-SP

\section{Janaina Benatti de Almeida', Jéssica Steffany Miranda", Sandra Cristina Shiguemi Miyasaki"', Silvio Fernando Guideti Marques"I' \\ 1, "Faculdade de Medicina de São José do Rio Preto (FAMERP). Programa de Residência Multiprofissional. São José do Rio Preto-SP, Brasil. \\ "Universidade Estadual Paulista (UNESP). Programa Mestrado Profissional em Enfermagem. Botucatu-SP, Brasil. III Centro Universitário de Lins (UNILINS). Curso de Enfermagem. Lins-SP, Brasil.}

\section{RESUMO}

O estudo objetivou conhecer a prevalência do tabagismo e descrever o perfil e hábitos tabágicos entre universitários de Lins-SP. Uma amostra de 368 alunos respondeu a um questionário com perguntas dirigidas a fumantes, ex-fumantes e não fumantes. A prevalência de tabagismo foi de $11,7 \% ; 85,3 \%$ dos alunos não eram fumantes e 4,0\% foram classificados como ex-fumantes. Do total de alunos entrevistados, 54,1\% eram mulheres. Foi observado consumo médio de 5,1 \pm 5,4 cigarros por dia, sendo que todos os universitários tabagistas utilizavam o cigarro industrializado. Este índice de prevalência assemelha-se aos de outros Centros Universitários e demanda políticas de controle do tabagismo nos meios universitários.

Descritores: Tabagismo; Hábito de fumar;Epidemiologia; Política de saúde.

\section{ABSTRACT}

The study aimed to know the prevalence of smoking and to describe the profile and smoking habits of undergraduate students of Lins-SP. A sample of 368 undergraduate students answered a questionnaire with questions directed at smokers, ex-smokers and nonsmokers The prevalence of smoking was of $11,7 \%$; about $85,3 \%$ of the students were nonsmokers and $4,0 \%$ were classified as ex-smokers. Of the students interviewed, 54,1\% were women. It was noted that the average use was from 5,1 to 5,4 cigarettes per day, and that the students used industrialized cigarettes. This index of prevalence correlates to the ones of others University Centers and demands politics of smoking control among the university environment.

Key words: Smoking; Smoking habits; Epidemiology; Health policy.

\section{RESUMEN}

El estudio tuvo como objetivo conocer la prevalencia del tabaquismo y describir el perfil y hábitos de tabaquismo entre estudiantes universitarios de Lins-SP. Una muestra de 368 estudiantes respondieron a un cuestionario con preguntas dirigidas a fumadores, ex fumadores y no fumadores. La prevalencia de tabaquismo fue de $11,7 \% ; 85,3 \%$ de los estudiantes eran no fumadores y $4,0 \%$ fueron clasificados como ex fumadores. Entre los encuestados, el 54,1\% eran mujeres. Fue observado un consumo medio de 5,1 \pm 5,4 cigarrillos por día, y todos los estudiantes fumadores utilizaban cigarrillos comerciales. Esta tasa de prevalencia es similar a otros centros universitarios y demanda políticas de control del tabaco en los círculos universitarios. Palabras clave: Tabaquismo; Hábitos de fumar; Epidemiologia; Política de Salud. 


\section{INTRODUÇÃO}

O tabagismo é, hoje, a principal causa de enfermidades evitáveis e incapacidades prematuras, e chegará a ser a primeira causa de morte evitável no século XXI ${ }^{(1)}$. É considerado uma pandemia silenciosa, uma vez que, a cada ano, morrem cerca de 4 milhões de pessoas em todo o mundo de doenças relacionadas ao tabaco. O consumo de 1 a 20 cigarros por dia tem sido associado ao aumento do risco de câncer de estômago ${ }^{(2)}$. O consumo maior que um maço por dia ( $>$ 20 cigarros/dia) eleva em 13 vezes o risco de ter câncer de cabeça e pescoço ${ }^{(3)}$.

Diversos estudos realizados em nosso país ${ }^{(4-5)}$ e no mundo $^{(6-7)}$ apontam que o hábito de fumar se instala precocemente, já que $80 \%$ dos atuais adultos fumantes declararam ter iniciado nesta prática antes dos dezoito anos de idade.

Estas informações confirmam a tendência mundial de aumento da prevalência do uso de cigarros entre a população de adolescentes e adultos jovens, principalmente entre os estudantes universitários, jovens estes considerados público com grande suscetibilidade de envolvimento com o tabaco ${ }^{(8)}$. Diante da gravidade desse quadro, vários estudos sugerem que medidas antitabágicas sejam direcionadas prioritariamente a esta população ${ }^{(9-10)}$.

Acredita-se que, se não forem tomadas medidas adequadas para o controle dessa pandemia, ao redor do ano de 2020, o tabagismo será o responsável por 10 milhões de mortes anuais, com proporções de uma em cada seis pessoas consumidoras de tabaco. Desses óbitos, 7 milhões ocorrerão nos países em desenvolvimento ${ }^{(1)}$.

Tendo em vista estes aspectos, este trabalho teve como objetivos avaliar a prevalência, conhecer as características do tabagismo, e servir como medida auxiliar para o desenvolvimento de estratégias que visem à profilaxia do consumo de cigarros na população universitária da região de Lins-SP.

\section{METODOLOGIA}

Realizou-se um estudo transversal com alunos de graduação devidamente matriculados durante o primeiro semestre de 2008 nos três Centros Universitários da cidade de Lins-SP. Para calcular o tamanho da amostra, utilizou-se a população total de 4.530 universitários matriculados e um erro amostral tolerável de $5 \%$, para possibilitar intervalo de confiança de $95 \%$. Dessa maneira, foi obtida, inicialmente, como amostra ideal para o desenvolvimento deste estudo, um grupo de 368 universitários ${ }^{(11)}$.

Para a obtenção da amostra, foi realizado sorteio aleatório simples entre os alunos de todos os 33 cursos oferecidos pelos três Centros Universitários. O número de alunos entrevistados por curso foi determinado através do produto entre o fator de proporcionalidade, que relaciona o tamanho da amostra com o tamanho da população estudada, e o número total de alunos do respectivo curso.

Os cursos que fizeram parte do estudo estão listados no Quadro 1.

\begin{tabular}{|c|c|}
\hline $\begin{array}{c}\text { CENTRO } \\
\text { UNIVERSITÁRIO }\end{array}$ & CURSOS \\
\hline $\begin{array}{l}\text { Centro } \\
\text { Universitário } 1\end{array}$ & $\begin{array}{ll}\text { - } & \text { Direito } \\
\text { - } & \text { Nutrição } \\
\text { Odontologia }\end{array}$ \\
\hline $\begin{array}{l}\text { Centro } \\
\text { Universitário } 2\end{array}$ & $\begin{array}{ll}\text { - } & \text { Tecnologia em Química } \\
& \text { Industrial } \\
\text { - } & \text { Tecnologia em Análise de } \\
& \text { Sistemas Desenvolvimento de } \\
& \text { Sistemas para a Internet } \\
\text { - } & \text { Sistemas de Informação } \\
\text { - } & \text { Engenharia de Automação } \\
\text { - } & \text { Engenharia Civil } \\
\text { - } & \text { Engenharia da Computação } \\
\text { - } & \text { Engenharia Ambiental } \\
\text { - } & \text { Engenharia Elétrica } \\
\text { - } & \text { Marketiatura em Informática } \\
\text { - Serviço Social } \\
\text { - Secretariado Executivo } \\
\text { - } \quad \text { Tdministração } \\
\text { - Gecnologia em Processos } \\
\text { - Enfenciais } \\
\text { Enfermagem }\end{array}$ \\
\hline $\begin{array}{l}\text { Centro } \\
\text { Universitário } 3\end{array}$ & $\begin{array}{ll}\text { - } & \text { Desenvolvimento de Sistemas } \\
\text { - } & \text { Quáa a Internet } \\
\text { - } & \text { Matemática } \\
\text { - } & \text { Ciências Contábeis } \\
\text { - } & \text { Psicologia } \\
\text { - } & \text { Administração } \\
\text { - } & \text { Pedtória } \\
\text { - } & \text { Letrasogia } \\
\text { - } & \text { Biologia } \\
\text { - } & \text { Enfermagem } \\
\text { - } & \text { Tducação Física } \\
\text { - } & \text { Fisioterapia }\end{array}$ \\
\hline
\end{tabular}

\section{Quadro 1 - Cursos dos Centros Universitários de Lins-SP que participaram do estudo.}

Para obtenção das informações necessárias sobre o consumo de tabaco, utilizou-se um questionário pré-codificado, individual e confidencial, com perguntas relacionadas ao hábito tabágico, distribuídas em três domínios, direcionadas aos fumantes regulares e fumantes ocasionais, aos ex-fumantes e aos não fumantes. Este questionário continha variáveis demográficas (idade, sexo e cor), socioeconômica (renda familiar em salários mínimos vigentes na época da pesquisa), variáveis relacionadas ao tabagismo (se é fumante, tipo de fumo, consumo diário, idade de início, tempo de tabagismo e, caso ex-fumante, tempo de tabagismo e de suspensão do fumo), tipo de tabaco (industrializado, confeccionado pelo próprio fumante, charuto e cachimbo), convivência com fumantes (pais, irmãos, amigos) 
e local de convivência (casa, universidade, trabalho). Também foram investigados o consumo de bebidas alcoólicas, prática de esportes e presença de sintomas como tosse seca, tosse produtiva e sons pulmonares ("chiado no peito").

O ponto de corte para a definição de fumante foi "ter fumado um ou mais cigarros por dia há pelo menos um mês", e para ex-fumantes "aqueles que, na ocasião da pesquisa, não eram fumantes, mas que já fizeram uso de cigarros em outra época".

O questionário foi aplicado pelos pesquisadores diretamente aos alunos após esclarecimentos e orientações básicas sobre os objetivos da pesquisa em questão, ficando o universitário livre para decidir sobre sua participação; o Termo de Consentimento Livre e Esclarecido (TCLE) foi assinado. O estudo, atendendo à Resolução no 196/1996, do Conselho Nacional de Saúde, foi aprovado pelo Comitê de Ética em Pesquisa com Seres Humanos e Animais (CEPHA) da Universidade de Marília - UNIMAR.

Foi utilizado o teste do qui-quadrado para avaliar a existência de associações entre as variáveis analisadas, sendo considerado o valor de $p<0,05$ para significância estatística.

\section{RESULTADOS E DISCUSSÃO}

Vários levantamentos epidemiológicos têm sido realizados no Brasil desde meados dos anos 80, descrevendo a prevalência e os fatores associados ao tabagismo entre os universitários, sendo que a maioria avaliou as características do tabagismo entre a população universitária pertencente à área da saúde.

A maior parte dos universitários (308) se declarou não-fumante, representando $83,7 \%$ da amostra. A prevalência de tabagismo foi de $11,7 \%$ (43), sendo $6,3 \%$ de fumantes regulares (23) e $5,4 \%$ de fumantes ocasionais (20). Como ex-fumantes, foram classificados $4,6 \%$ dos alunos (17).

Em relação à distribuição da amostra segundo o sexo, não foi encontrada diferença estatisticamente significativa $(p<$ $0,05)$, uma vez que $54,1 \%$ dos entrevistados eram do sexo feminino, e dentre os universitários tabagistas, $53,5 \%$ pertenciam a este gênero.

Dentre os estudantes classificados como não fumantes, $58,4 \%$ conviviam com fumantes, sendo esta convivência estratificada na Tabela 1. Fica demonstrado que mais da metade dos não fumantes são tabagistas passivos. O tabagismo passivo está relacionado com a inalação da fumaça de derivados do tabaco por indivíduos não fumantes, que convivem com fumantes em ambientes fechados. Esta fumaça traz efeitos mais graves ao organismo, traduzidos por uma incidência 30\% maior de câncer pulmonar e aumento de $24 \%$ de infarto do miocárdio.

\section{Tabela 1 - Distribuição dos alunos não fumantes de acordo} com a convivência com fumantes.

\begin{tabular}{lcc}
\hline Convivência com Fumantes & N & \% \\
\hline Universidade & 81 & 45,0 \\
Domicílio & 58 & 32,2 \\
Trabalho & 41 & 22,8 \\
TOTAL & 180 & 100,0 \\
\hline
\end{tabular}

Com relação aos estudantes que se declararam ex-fumantes, os motivos principais citados para a interrupção deste hábito foram: força de vontade $(88,2 \%)$, conscientização dos malefícios do tabagismo (5,9\%) e problemas de saúde (5,9\%).

Quanto à prevalência de tabagistas nos três Centros Universitários estudados, os resultados obtidos encontram-se dentro dos parâmetros de outros estudos ${ }^{(4-11)}$, exceto, isoladamente, o Centro Universitário 1, o qual apresentou uma prevalência $10 \%$ maior do que o esperado. Porém, o número total de fumantes $(11,7 \%)$ encontra-se de acordo com estudos prévios do assunto (Quadro 2).

\begin{tabular}{|l|c|c|}
\hline Instituição & N & \% \\
\hline Centro Universitário 1 & 7 & 21,2 \\
\hline Centro Universitário 2 & 16 & 10,5 \\
\hline Centro Universitário 3 & 20 & 11,0 \\
\hline
\end{tabular}

\section{Quadro 2 - Prevalência de tabagismo nos três Centros Universitários estudados.}

Alguns estudos têm demonstrado que as prevalências de tabagismo entre estudantes de cursos da área de saúde são inferiores aos resultados encontrados entre alunos de outros cursos superiores $^{(7-9)}$. Em nosso estudo, entretanto, observou-se inversão neste contexto, uma vez que a área de ciências biológicas e da saúde apresentou a maior prevalência de tabagismo $(12,6 \%)$, seguida pela área de humanas $(10,4 \%)$ e de exatas $(10,0 \%)$.

Em outro estudo, foi relatado que mais de $50 \%$ dos estudantes tabagistas regulares tendiam a aumentar o consumo tabágico após a admissão ao ambiente universitário( ${ }^{(9)}$. Mesmo frente a esta tendência de crescimento do tabagismo entre universitários em âmbito internacional, no Brasil podemos observar o oposto, mediante estudos que avaliaram a tendência tabágica entre os alunos das principais universidades brasileiras, que apontam para uma tendência de redução do tabagismo entre os universitários nas últimas décadas ${ }^{(12)}$. Resultado semelhante foi observado em nosso estudo, onde a prevalência de tabagismo entre os universitários da região de Lins foi de $11,7 \%$.

Com relação aos cursos, individualmente, observou-se que as maiores prevalências de tabagismo foram encontradas nos cursos de Engenharia Civil, do Centro Universitário 2, e Química e Terapia Ocupacional, ambos do Centro Universitário 3, com $40,0 \%$ de fumantes. Outros cursos que apresentaram porcentagens elevadas de estudantes tabagistas foram Direito, do Centro Universitário 1 (33,3\%), Tecnologia em Sistemas para Internet e Engenharia Elétrica, do Centro Universitário $2(25,0 \%$ e $22,2 \%$, respectivamente) e História, do Centro Universitário 3 (20\%).

No Quadro 3 estão reunidas as porcentagens de tabagistas de cada curso dos três Centros Universitário de Lins-SP. Os cursos que não apresentaram nenhum indivíduo entrevistado como fumante foram: Engenharia da Computação, Licenciatura em Informática, Secretariado Executivo, Serviço Social, Sistemas de Informação, Tecnologia em Análise de Sistemas, 


\begin{tabular}{|l|c|c|l|c|c|l|c|c|}
\hline \multicolumn{2}{|c|}{ Centro Universitário 1 } & \multicolumn{4}{|c|}{ Centro Universitário 2 } & \multicolumn{3}{c|}{ Centro Universitário 3 } \\
\hline Curso & $\mathrm{N}$ & $\%$ & Curso & $\mathrm{N}$ & $\%$ & Curso & $\mathrm{N}$ & $\%$ \\
\hline Direito & 05 & 33,3 & Engenharia Civil & 04 & 40,0 & Química & 04 & 40,0 \\
\hline Odontologia & 02 & 15,4 & $\begin{array}{l}\text { Tecnologia em Sistemas } \\
\text { para Internet }\end{array}$ & 01 & 25,0 & Terapia Ocupacional & 02 & 40,0 \\
\hline & & Engenharia Elétrica & 02 & 22,2 & Psicologia & 02 & 18,2 \\
\hline & & & Enfermagem & 02 & 18,2 & Enfermagem & 03 & 17,6 \\
\hline & & & Engenharia Ambiental & 02 & 15,4 & Letras & 02 & 14,3 \\
\hline & & Engenharia de Automação & 03 & 12,0 & $\begin{array}{l}\text { Tecnologia em Sistemas } \\
\text { para Internet }\end{array}$ & 01 & 11,1 \\
\hline & & & Administração & 01 & 10,0 & Biologia & 01 & 10,0 \\
\hline & & Marketing & 01 & 8,3 & Educação Física & 02 & 8,0 \\
\hline & & & & & & Fisioterapia & 01 & 6,3 \\
\hline
\end{tabular}

Quadro 3 - Prevalência de tabagismo nos cursos de cada Centro Universitário de Lins-SP.

Tecnologia em Processos Gerenciais e Tecnologia em Química Industrial do Centro Universitário 2; Administração, Ciências Contábeis, Matemática e Pedagogia do Centro Universitário 3 e Nutrição do Centro Universitário 1.

Um fator que merece atenção em estudos de prevalência de tabagismo é o tipo de tabaco mais utilizado. Em alguns trabalhos, mais da metade dos universitários tabagistas norte-americanos utilizava cigarro industrializado ${ }^{(8)}$. Segundo outros autores, este tipo de tabaco é o mais consumido entre os universitários, seguido por cachimbo e charuto ${ }^{(7)}$. Todos os fumantes deste estudo declararam utilizar cigarros comercializados.

Nos dados referentes à idade dos tabagistas entrevistados, observou-se as seguintes prevalências: de 21 a 23 anos - 53,5\%, 18 a 20 anos $-30,2 \%, 24$ a 26 anos e $\geq 30$ anos com $7,0 \%$ cada uma, seguida por de 27 a 29 anos com 2,3\%.

Sobre a renda familiar (em salários mínimos) dos tabagistas, mais da metade $(53,5 \%)$ tem renda entre 4,0 e 7,0 salários, seguido por 1,5 a 3,9 e mais de 7,0 salários ambos com percentual de $23,2 \%$ dos entrevistados. Dos tabagistas $58,1 \%$ vivem com os pais; $25,6 \%$ com amigos e/ou outros universitários; $11,6 \%$ sozinhos e 2,3\% com cônjuge e a mesma proporção com filhos.

Quanto ao número de cigarros consumidos por dia, em nosso estudo verificamos que a maior parte dos tabagistas entrevistados $(51,2 \%)$ fuma entre 1 a 5 cigarros por dia (Tabela 2 ).

Tabela 2 - Número de cigarros consumidos por dia pelos tabagistas dos três Centros Universitários.

\begin{tabular}{ccc}
\hline Número de cigarros por dia & $\mathbf{N}^{\circ}$ & $\%$ \\
\hline 1 a 5 & 22 & 51,2 \\
6 a 10 & 13 & 30,2 \\
11 a 15 & 0 & 0,0 \\
16 a 20 & 04 & 9,3 \\
> 20 & 04 & 9,3 \\
TOTAL & 43 & 100,0 \\
\hline
\end{tabular}

Em relação há quanto tempo o indivíduo é fumante, as maiores prevalências foram de $41,9 \%$ e $23,2 \%$ para o tempo de 1 a 3 anos e de 4 a 6 anos, respectivamente (Tabela 3). Em nosso estudo pode-se observar que quanto maior o tempo de fumo, maior o número de cigarros por dia.

\section{Tabela 3 - Tempo de fumo dos tabagistas nos três Centros Universitários estudados.}

\begin{tabular}{ccc}
\hline Tempo de fumo & $\mathbf{N}^{\mathbf{0}}$ & $\mathbf{\%}$ \\
\hline Menos de 1 ano & 04 & 9,3 \\
1 a 3 anos & 11 & 25,6 \\
4 a 6 anos & 18 & 41,9 \\
Mais de 7 anos & 10 & 23,2 \\
TOTAL & 43 & 100,0 \\
\hline
\end{tabular}

Com relação à idade de início no tabagismo, observou-se prevalência de $16,3 \%$ para o intervalo de 10 a 14 anos, $69,8 \%$ para o período de 15 a 19 anos e 13,9\% dos fumantes tinham entre 20 a 24 anos quando iniciaram a prática tabágica. Estes números mostram que, como em vários estudos ${ }^{(1-4)}$, a idade para início do tabagismo é, na maior parte dos casos, antes dos 18 anos.

Cerca de $67,4 \%$ dos pesquisados fumam mesmo estando doentes, enquanto $32,6 \%$ deixam o cigarro durante este período. Já $58,1 \%$ admitem que o cigarro lhe faça mal, enquanto $41,9 \%$ acreditam que não.

Quando questionados sobre o aconselhamento de profissionais de saúde para deixar o hábito tabágico, mais da metade $(58,1 \%)$ afirmou que já recebeu este tipo de orientação; 90,7\% afirma ser capazes de deixar de fumar; $83,7 \%$ pretendem cessar o hábito tabágico e 46,5\% tentaram parar de fumar nos últimos doze meses. Observa-se que a grande maioria dos tabagistas admite ser capaz de cessar o hábito 
tabágico, subestimando assim, sua dependência do cigarro.

Outro ponto, ainda, que merece destaque é o consumo de bebidas alcoólicas, pois, além de causar inúmeras doenças, quando do seu uso combinado ao tabaco, somam-se os efeitos nocivos deste último, além do alcoolismo ser fator dificultador para a interrupção do tabagismo ${ }^{(9)}$. Acerca desta problemática, uma pesquisa realizada em Porto Alegre constatou que, entre alcoolistas, a prevalência de fumantes $(67 \%)$ é maior que entre os não alcoolistas (43\%). Isto indica que quem bebe fuma mais, o que está de acordo com nossa pesquisa, haja vista que $74,4 \%$ associam o uso do tabaco à ingestão de álcool.

Dos motivos que levaram o indivíduo a fumar, a maioria $(44,2 \%)$ afirmou ter sido a influência dos amigos, 32,6\% por vontade própria e 7,0\% por modismo. A mesma porcentagem de 4,6\% para curiosidade, influência dos pais e por decepções vividas. E, por fim, 2,3\% para emagrecer.

Com o objetivo de se evitar o início e auxiliar na cessação do tabagismo, além de possibilitar a interrupção da convivência passiva dos não fumantes com os fumantes, várias organizações internacionais, como a American College of Health Association e a American Cancer Society, têm recomendado que as universidades proíbam a venda, a publicidade e a distribuição de amostras gratuitas de produtos derivados do tabaco nos campi universitários, além de vetar o uso do tabaco em todos os locais da instituição ${ }^{(13)}$. No Brasil, a Política de Controle do Tabagismo inclui vigilância, legislação e incentivos econômicos, além de educação em escolas, locais de trabalho e nas unidades de saúde. Como passos necessários, o programa identifica: evitar a dependência, em especial entre crianças e adolescentes, promover ações para estimular a cessação, proteger os não fumantes dos perigos da fumaça ambiental do tabaco e promover a redução dos danos causados pelo tabaco, através de medidas de regulamentação do produto ${ }^{(14-15)}$. Tais estratégias têm sido intensificadas, entretanto, é necessário um estudo que avalie a sua eficácia em determinadas faixas de idade, em especial para jovens ${ }^{(16)}$.

\section{CONSIDERAÇÕES FINAIS}

A prevalência de tabagismo entre os universitários estudados é semelhante à prevalência encontrada em estudos de anos anteriores, o que demonstra que as medidas antitabágicas já existentes não estão sendo eficazes ou estão deixando de ser implementadas, visto que as proporções de tabagistas neste meio poderiam regredir.

Mais de $80 \%$ dos tabagistas de nosso estudo iniciaram o hábito antes dos 19 anos, ou seja, encontravam-se no início do período universitário ou final do ensino médio. Portanto, é imprescindível a tomada de medidas preventivas mais eficazes, como promover ações educativas e vetar o uso do tabaco em todos os locais da instituição universitária, evitando assim também o tabagismo passivo.

O aconselhamento profissional sobre os malefícios decorrentes do tabaco faz-se importante instrumento na luta contra o tabagismo. De acordo com nosso estudo, quase metade dos fumantes nunca receberam orientações sobre o hábito tabágico, o que demonstra pouco empenho dos profissionais da área, indicando uma subestimação do problema.

É, portanto, importante o período universitário, quanto à aplicação de medidas profiláticas na cessação do fumo e não aderência a este hábito, trazendo, por conseguinte, um índice menor de morbimortalidade relacionado ao tabaco.

\section{REFERÊNCIAS}

1. Malcon MC, Menezes AMB, Chatkin M. Prevalência e fatores de risco para tabagismo em adolescentes. Rev. Saúde Pública. 2003 Fev; 37(1): 1-7.

2. Hoshiyama Y, Sasaba T. A case-control study of stomach cancer and its relation to diet, cigarettes, and alcohol consumption in Saitama. Cancer Causes Control. 1992 Set; 3(5): 441- 8 .

3. Dedivitis RA, França CM, Mafra ACB, Guimarães FT, Guimarães AV. Características clínico-epidemiológicas no carcinoma espinocelular de boca e orofaringe. Rev. Bras. Otorrinolaringol. 2004 Jan; 70(1): 35-40.

4. Andrade APA, Bernardo ACC, Viegas CAA, Ferreira DBL, Gomes TC, Sales MR. Prevalência e características do tabagismo em jovens da Universidade de Brasília. J. Bras. Pneumol. 2006 Fev; 32(1): 23-28.

5. Instituto Nacional do Câncer. Ação global para o controle do tabaco. Primeiro Tratado Internacional de Saúde Pública. Brasília: Ministério da Saúde; 2002. [citado em: 15 set 2005]. Disponível em: http://dtr2001.saude.gov.br/ bvs/publicacoes/inca/acao_global.pdf.

6. Moskal PD, Dziuban CD, West GB. Examining the use of tobacco on college campuses. J. Am. Coll. Health. 1999 Maio; 47(6): 260-5.

7. Haddad LG, Malak MZ. Smoking habits and attitudes towards smoking among university students in Jordan. Int. J. Nurs. Stud. 2002 Nov; 39(8): 793-802.

8. Rigotti NA, LJE, Wechsler Henry. US college students use of tobacco products: results of a national survey. Jama. 2000 Ago; 284(6): 699-705.

9. Baska TSS, Mad'ar R. Smoking habits in university students in Slovakia. Cent Eur J Public Health. 2000 Nov; 8(4): 245 - 248. 
10. Halperin AC, Rigotti NA. US public universities' compliance with recommended tobacco-control policies. J. Am. Coll. Health, 2003 Mar; 51(5): 181-188.

11. Oliveira Ely Francina Tannuri de, Grácio Maria Cláudia Cabrini. Análise a respeito do tamanho de amostras aleatórias simples: uma aplicação na área de Ciência da Informação. Rev. Ciência Informação. 2005 Jun; 6 (3).

12. Menezes A, Palma E, Holthausen R, Oliveira R, Oliveira PS, Devens E, Steinhaus L, Horta B, Victora CG. Smoking time evolution among medical students, 1986, 1991, 1996. Rev Saude Publica. 2001 Abr; 35(2): 165-169.

13. American Cancer Society. Smoke-free New England campus initiative, seven step policy plan. American Cancer Society. [cited 2005 Jul 28]. Available from: http://www.cancer.org/ docroot/com/content/div_ne/com_4_2x_smoke-free_new england.asp? sitearea $=$ com

14. Banco Mundial; Saúde, Nutrição e População. Controle do Tabagismo no Brasil. Brasil; 2007.

15. Hortense FTP, Carmagnani MIS, Brêtas ACP. O significado do tabagismo no contexto do câncer de laringe. Rev Bras Enferm 2008 Fev; 61(1): 24-30.

16. Silva AO, Sousa CMM, Gaspar MFM, Paredes MAS, Tura LFR, Jesuíno JC. Tabaco e saúde no olhar de estudantes universitários. Rev Bras Enferm 2008 Ago; 61(4): 423-427. 